\title{
Measurements and modelling of dendritic growth velocities of pure Fe with thermoelectric magnetohydrodynamics convection
}

Rijie Zhao ${ }^{\mathrm{a}}$, Jianrong $\mathrm{Gao}^{\mathrm{a}, *}$, Andrew Kao ${ }^{\mathrm{b}}$, Koulis Pericleous ${ }^{\mathrm{b}}$

${ }^{a}$ Key Laboratory of Electromagnetic Processing of Materials (Ministry of Education), Northeastern University, Shenyang 110819, China

${ }^{\mathrm{b}}$ Centre for Numerical Modelling and Process Analysis, University of Greenwich, London SE10 9LS, United Kingdom

\begin{abstract}
Dendritic growth velocities of pure Fe under static magnetic fields of intensity ranging from $B=0 \mathrm{~T}$ to $B=6 \mathrm{~T}$ were measured using a high speed camera. The data measured at undercoolings up to $\Delta T=190 \mathrm{~K}$ show a depression followed by a recovery of the growth velocities as the magnetic field intensity increased from a low range, $B=1 \sim 3$ T to a high range, $B=4 \sim 6 \mathrm{~T}$. These magnetic field effects are similar to those previously observed for pure $\mathrm{Ni}$ and can be attributed to competing thermoelectric magnetohydrodynamic (TEMHD) convection patterns in the local liquid. The experimental measurements for the two metals were modelled using a threedimensional dendritic growth theory taking into account convection to estimate the effective flow velocities in the tip growth direction. The calculated effective flow velocities identify two undercooling dependences and a distinct type of magnetic field intensity dependence in common for the two metals. In comparison, the calculated effective flow velocities for pure Fe are generally smaller in magnitude. This difference between the two metals can be related to their differences in material-dependent properties as is revealed by a simple model proposed for a transverse TEMHD flow.
\end{abstract}

Keywords: A1. Dendrites; A1. Solidification; A1. Magnetic fields; A1. Convection; A2. 
Growth from melt; B1. Metals.

* Corresponding author. Tel.: +86-24-83681915; fax: +86-24-83681758. E-mail address:jgao@mail.neu.edu.cn (J. Gao). 


\section{Introduction}

Thermoelectric currents are formed within a conducting material, of which a local thermal gradient and an absolute thermoelectric power gradient are not parallel to each other. When a static magnetic field is applied, it acts on these currents generating a Lorentz force. This Lorenz force drives thermoelectric magnetohydrodynamic (TEMHD) convection in the liquid state of the material, which transports heat and in turn alters the local thermal gradient $[1,2]$. The theory of TEMHD convection was proposed by Shercliff [3] in 1970's. A TEMHD effect on the quality of single crystals or directionally solidified polycrystals was observed by several groups later [4-11]. It was shown that strong TEMHD convection can be driven by a magnetic field of less than $B=1$ T causing changes of chemical segregation patterns of the grown crystals. Moreover, TEMHD convection alters the morphology of the solid-liquid interface in directional solidification by promoting a cellular-to-dendritic transition $[12,13]$. Such findings have aroused much research interest in TEMHD phenomena in various solidification processes, from both fundamental and technical perspectives [14-19].

In free solidification, the thermal gradient around a dendritic tip is generally higher than that achieved in single crystal growth or directional solidification. As a result, TEMHD convection can be stronger in free dendritic growth than in constrained dendritic growth. Kao $[1,14,15]$ investigated TEMHD convection in free dendritic growth and its influence on the morphology and tip growth kinetics of dendrites differently oriented with respect to a static magnetic field using numerical modelling. His work unveiled three kinds of flow patterns due to TEMHD: a transverse flow, an incident flow and a circular flow. Fig. 1 provides a representative TEMHD around an equiaxed dendrite highlighting key features [2]. A magnetic field parallel to the tip growth direction ( $z$ direction) leads to a circular flow around the dendritic tip, and a 
magnetic field perpendicular to the tip growth direction leads to a circulation over the tip. The latter behaves likes a transverse flow in the vicinity of the tip and will be the focus of the paper. The incident flow that promotes growth is a much more complicated situation and not shown in Fig. 1. Gao et al. [20] investigated dendritic growth of pure Ni under static magnetic fields experimentally. They found that the dendritic growth velocities for undercoolings up to $\Delta T=120 \mathrm{~K}$ are depressed at low magnetic field intensity of $B=1 \sim 5 \mathrm{~T}$ and recover at a high magnetic field intensity of $B=6 \mathrm{~T}$. They interpreted such magnetic field effects by considering the competition of the three kinds of TEMHD flows, which can promote or depress tip growth velocities depending on its kind. They also showed that the tip growth velocities of pure Ni can be modelled using a three-dimensional dendritic growth theory with convection proposed by Alexandrov and Galenko (AG theory in short) $[21,22]$. In their modelling, a constant flow velocity was assumed for a given magnetic field intensity. This assumption is not realistic because the velocities of TEMHD flows depend not only on magnetic field intensity, $B$, but also on undercooling and material properties $[13,14]$. Thus, it is necessary to use refined modelling of the measured dendritic growth velocities of pure Ni to understand dependences of TEMHD flows on magnetic field intensity and on undercooling. On the other hand, there is currently a paucity of information in the literature on the dependence of TEMHD flows on material properties [24], especially in undercooled conditions. For these two reasons, dendritic growth velocities of pure Fe under static magnetic fields were measured in the present work and compared with the data of pure $\mathrm{Ni}[20]$. The measured data of both metals were modelled using the AG theory $[21,22]$ to calculate effective flow velocities driven by TEMHD convection in the direction of primary tip growth. The modelling not only demonstrated universal dependences of effective flow velocities on undercooling and magnetic field intensity, but also 
highlighted the differences in the effective flow velocities between the two metals. In this paper, several different velocities are considered. For clarity, we explicitly state their meanings here. $V$ is the tip growth velocity, $U$ is the real flow velocity, $U_{\text {eff }}$ is an effective flow velocity at the dendritic tip as described in the AG theory, and $U_{T E}$ is the modelled transverse flow velocity used in an analytical solution derived in Section 5.

\section{Experimental}

A sphere-like sample of electrolytic Fe (99.99\% purity) with a mass of $1 \mathrm{~g}$ was used for measurements of dendritic growth velocities. In the measurements, the sample was inductively heated and was undercooled by the glass fluxing treatment. In melting and solidification, static magnetic fields of intensity ranging from $B=1 \mathrm{~T}$ to $B=6 \mathrm{~T}$ were imposed on the sample using a superconducting magnet. The sample was melted and solidified more than 20 times under each magnetic field intensity to acquire a wide spectrum of undercooling. Crystal nucleation occurred stochastically on the lower surface of the sample, which was in intimate contact with fluxed glass. No triggering of crystal nucleation was attempted in the measurements because of small space available in the bore of the superconducting magnet. In each cycle of melting and solidification, a single-colour pyrometer with a wavelength of $0.9 \mu \mathrm{m}$ was used to measure the surface temperature of the sample at a sampling rate of $100 \mathrm{~Hz}$. During solidification, a high-speed camera was used to monitor and record recalescence processes of the sample at a frame rate of $87,600 \mathrm{fps}$. To isolate the effects of TEMHD convection, the measurements were also performed without any magnetic field. More details of the experimental set-up and procedures can be found elsewhere [20,24].

The measured surface temperature of the sample was calibrated by taking into account a macroscopic thermal gradient to determine undercooling of the sample [24]. The 
recorded videos were analyzed using a Matlab-based computer program to determine growth velocities of dendritic tips in the undercooled liquid. The analysis method is illustrated in Fig. 2. In recalescence, a thermal front due to diffusion of released latent heat was assumed to travel like a spherical wave from a nucleation site towards the other side of the sample surface at a constant speed. The nucleation site could be determined by matching the loci of the thermal front recorded in the videos with the spherical wave transmitting from it. Then, the traveling distance of the thermal front away from it was calculated as a function of time by tracking how far the thermal front moved over successive frames from the high-speed camera. A linear law was fitted to the distance versus time relationship, and its slope was taken as the traveling speed of the thermal front (see the right panel of Fig. 2). Because the thermal diffusion distance is shorter than the length of a single-pixel of the high-speed camera $(100 \mu \mathrm{m})$, the traveling speed of the thermal front gives a good approximation of dendritic growth velocity in a recalescence event [25]. The thermal front is zig-zagged at low undercoolings and becomes smooth at high undercoolings. Thus, the primary source of errors is from the spatial and the temporal resolution at low and high undercoolings, respectively. However, the latter are generally smaller as the high frame rate of the high speed camera can sufficiently capture frames of the order $10^{1}$ even at high undercoolings where the dendritic growth velocity may reach an order of $10^{2} \mathrm{~m} / \mathrm{s}$ of magnitude. Therefore, the errors are smaller for higher tip growth velocities. To minimize the errors, each video was analyzed three times. The standard deviations of the measured growth velocities are typically smaller than $5 \%$ of an averaged value.

\section{Results}

\subsection{Dendritic growth velocities without static magnetic fields}


Fig. 3 shows the measured data of pure $\mathrm{Fe}$ at $B=0 \mathrm{~T}$. The previous data of pure Ni at $B=0 \mathrm{~T}[15]$ is also shown for comparison. The growth velocities of pure Fe are generally lower than those of pure $\mathrm{Ni}$ at all accessed undercoolings (pure Fe: $\Delta T=53$ $\sim 307 \mathrm{~K}$; pure Ni: $\Delta T=32 \sim 211 \mathrm{~K})$. It is known that two partial undercoolings, thermal and kinetic undercoolings, determine crystal growth velocities at low and high undercoolings, respectively [26]. Thus, the smaller growth velocities of pure Fe can be ascribed to smaller thermal diffusivity and a lower interfacial kinetic coefficient of pure Fe $[27,28]$. A previous study showed that bulk flow in the glass-fluxed sample of pure Ni has a maximum flow velocity of $0.015 \mathrm{~m} / \mathrm{s}$ due to a reduction of the heating current and viscous damping in cooling [24]. The bulk flow in the glass-fluxed sample of pure Fe was assumed to have the same magnitude in its undercooled stage for the same reasons. This maximum flow velocity is by a factor of 19 smaller than the magnitude of a dendritic growth velocity of $0.288 \mathrm{~m} / \mathrm{s}$ of pure $\mathrm{Fe}$ at the lowest undercooling of $\Delta T$ $=53 \mathrm{~K}$. It is therefore assumed to have a negligible effect on the measured growth velocities of pure Fe. Under this hypothesis, the measured growth velocities of pure Fe was modelled using the AG theory $[21,22]$ by assuming a zero flow velocity. Table 1 lists the values of material properties used in the modelling. Among those properties, the stability selection constant $\sigma_{0}$ was set to be adjustable because its value is not available in literature. Using a value of $\sigma o=0.0097$, an excellent agreement was achieved between the measured growth velocities of pure Fe and the AG theory. Similar modelling was already performed for the data of pure Ni assuming $\sigma_{0}=0.191$ [24]. Dendritic tip radii could not be measured for either metal due to opaqueness of metallic melts, but could be calculated by the modelling. As shown in Fig. 3b, the dendritic tip radii of pure $\mathrm{Fe}$ are by one order of magnitude larger than those of pure $\mathrm{Ni}$ at all undercoolings. This difference can be understood by considering the smaller growth 
velocities and a smaller stability selection constant of pure Fe.

\subsection{Dendritic growth velocities under static magnetic fields}

As plotted in Fig. 4, the measured dendritic growth velocities of pure Fe under the static magnetic fields show a similar dependence on undercooling. They also show discernible variations with rising magnetic field intensity. However, it is not easy to observe the magnetic field dependence due to a scatter of the measured data. For this reason, a series of power laws of $V=a \cdot \Delta T^{b}$ were fitted to the data. Table 2 lists the two fitting parameters, correlation coefficient $R_{\text {corr }}$ and root-mean-square deviations of the data from the fitted curves as percentages. The power law fittings were acceptable because the correlation coefficients $R_{\text {corr }}$ are generally larger than 0.80 . Meanwhile, the root-mean-square deviations vary between $24 \%$ and $38 \%$ for different magnetic fields and have an averaged value of $32 \%$. Such large deviations can be attributed to a scatter of nucleation sites. In the present measurements, the location of the nucleation sites determined an angle of the tip growth direction with respect to the direction of the magnetic fields. The angle is known to affect the interaction between TEMHD convection and dendritic growth $[15,20]$ and causes the scatter of the data. As listed in Table 1, the fitting parameters $a$ and $b$ both show a strong dependence on magnetic field intensity. This dependence suggests that the static magnetic fields have significant effects on the dendritic growth velocities of pure Fe. To evaluate the effects of the static magnetic fields quantitatively, the fitted growth velocities of Fig. 4 were used instead of the measured data. As explained in Section 3.1, the dendritic growth velocities measured without the static magnetic fields can be used as the zero-flow growth velocities for the quantization of the magnetic field effects. For comparison, they were also fitted using a power law. The fitting parameters are listed in Table 2. The fitted growth velocities under each magnetic field intensity were reduced with respect to the 
zero-flow tip growth velocities up to a critical undercooling of $\Delta T=190 \mathrm{~K}$. At this critical undercooling, the variations of the reduced growth velocities with rising magnetic field intensity became comparable to the averaged scatter of $32 \%$ in the measured data. For convenience, this critical value will be referred to as the terminating undercooling thereafter. As shown in Fig. 5a, the reduced growth velocities of pure Fe are depressed at lower magnetic field intensity of $B=1 \sim 3 \mathrm{~T}$ and recover at higher magnetic field intensity of $B=4 \sim 6 \mathrm{~T}$. Such variations of the reduced growth velocities can also be attributed to the three kinds of TEMHD flow patterns in the undercooled liquid, which compete with each other and cause a change of the dendritic growth kinetics of pure Fe by affecting heat transport ahead of the growing dendrites $[14,15]$. The effects of the static magnetic fields on dendritic growth velocities of pure Ni were ever examined in a previous study [20]. However, the power-fitted growth velocities of pure Ni were reduced with respect to their maxima at each magnetic field intensity. More critically, the undercooling of the Ni sample was not calibrated by taking into account the macroscopic thermal gradient [25]. For these two reasons, the previous growth velocity data of pure $\mathrm{Ni}$ were re-examined here for comparison with those of pure Fe. They were also fitted by power laws. The fitting parameters are listed in Table 3. The fitted growth velocities were reduced with respect to those fitted to the measured data at $B=0 \mathrm{~T}$ up to a terminating undercooling of $\Delta T=120 \mathrm{~K}$. The reduced growth velocities are plotted in Fig. $5 \mathrm{~b}$ as a function of magnetic field intensity. They show a depression and a recovery at lower and higher magnetic field intensity, respectively. Such effects of the static magnetic fields are unchanged with respect to those shown in the previous study [20] and are similar to those on pure Fe. However, the reduced growth velocities of pure $\mathrm{Ni}$ at undercoolings ranging from $\Delta T=30 \mathrm{~K}$ to $\Delta T=70 \mathrm{~K}$ are raised above the zero-flow growth velocities at the highest magnetic field intensity of 
$B=6 \mathrm{~T}$. This "overshooting" effect was not justified in the previous study [24]. It is in sharp contrast to a weak effect of the same magnetic field intensity on pure Fe. There are other differences between the two metals. One difference is that the reduced growth velocities of pure $\mathrm{Ni}$ at $\Delta T=30 \mathrm{~K}$ are less depressed at the lower magnetic field intensity of $B=1 \sim 3 \mathrm{~T}$. Another difference is that the magnetic field effect on pure $\mathrm{Ni}$ becomes indiscernible from the scatter of the experimental data at a lower terminating undercooling than that for pure $\mathrm{Fe}(\Delta T=120 \mathrm{~K}$ for $\mathrm{Ni}$ vs $\Delta T=190 \mathrm{~K}$ for $\mathrm{Fe})$. The similarity and differences between the two metals can be attributed to universal and material-dependent characteristics of TEMHD flows, respectively as was revealed by modelling the reduced growth velocities shown below.

\section{Modelling of growth velocity with TEMHD flows}

The AG theory was shown to provide a consistent explanation of dendritic growth velocities and tip radii of succinonitrille dendrites observed under different flow conditions bound to the levels of gravity [20]. Thus, its capability of predicting threedimensional growth of an equiaxed dendrite with convection was justified. In this theory, the convective flow is set to be along the direction of primary tip growth. In order to estimate TEMHD flow velocities using this theory, we assume here that the three kinds of TEMHD flows can be simplified into two kinds of equivalent flows. The transverse flow slows down primary tip growth velocities and thus, can be represented by an outgoing flow in the direction of primary tip growth. The circulating flow coarsens primary tips and can be represented by an outgoing flow as well. The incident flow can be accepted as it is because it transports the undercooled liquid towards the primary tips and promotes tip growth. To distinguish from those of the true TEMHD flows $U$, the velocities of the two equivalent flows are termed as effective flow 
velocities and denoted as $U_{\text {eff. }}$ To ease a mathematical treatment, a positive and a negative sign is assigned to the velocities of the incident flow and the outgoing flow, respectively. With such simplifications, $U_{\text {eff }}$ at dendritic tips of pure Fe and pure Ni were determined by matching the modelled growth velocities to the power-law fitted growth velocities of Fig. 4. The parameters used in the modelling are the same as those listed in Table 1.

The calculated $U_{\text {eff }}$ values for pure Fe are plotted in Fig. 6. They show a similar undercooling dependence at the lower magnetic field intensity of $B=1 \sim 3 \mathrm{~T}$. In this dependence, the magnitude of $U_{\text {eff }}$ increases with rising undercooling. There are a few humps, which can be attributed to the numerical errors in determining the effective flow velocities using the AG theory. The undercooling dependence of $U_{\text {eff }}$ becomes weaker at the higher magnetic field intensity and shows a minor change at $B=5 \mathrm{~T}$. A negative maximum of $U_{\text {eff }}=-1.72 \mathrm{~m} / \mathrm{s}$ merges at $\Delta T=150 \mathrm{~K}$. After reaching the maximum, the magnitude of $U_{\text {eff }}$ values shows an opposing trend with rising undercooling. However, the $U_{\text {eff }}$ values keep their negative sign for undercoolings up to $\Delta T=190 \mathrm{~K}$. At the highest magnetic field intensity of $B=6 \mathrm{~T}$, the $U_{\text {eff }}$ values show a second type of undercooling dependence. They possess a positive sign for undercoolings up to $\Delta T=$ $110 \mathrm{~K}$ and reach a maximum of $U_{\text {eff }}=0.05 \mathrm{~m} / \mathrm{s}$ at $\Delta T=90 \mathrm{~K}$. Following the change of their sign from positive to negative, their magnitude shows a steady increase with rising undercooling. As seen in Fig. 6b, the $U_{\text {eff }}$ values for pure Fe show a similar magnetic field intensity dependence for undercoolings up to $\Delta T=190 \mathrm{~K}$. Their magnitude increases with rising magnetic field intensity first. After reaching a negative maximum at $B=2 \mathrm{~T}$, it shows a declining tendency. Its declining slows down at the magnetic field intensity of $B=5 \mathrm{~T}$. The magnitude of the negative maximum is enlarged with rising undercooling but the enlargement becomes faster for $\Delta T>150 \mathrm{~K}$. 
The $U_{\text {eff }}$ values for pure Ni are plotted in Fig. 7a as a function of undercooling. They show a similar undercooling dependence in a range of magnetic field intensity from $B$ $=1 \mathrm{~T}$ to $B=5 \mathrm{~T}$. The trend in this dependence is similar to that observed for pure Fe, but is extended to a higher magnetic field intensity ( $B=5 \mathrm{~T}$ for pure Ni vs $B=3 \mathrm{~T}$ for pure $\mathrm{Fe}$ ). A second type of undercooling dependence was observed at $B=6 \mathrm{~T}$. It is similar to that observed for pure Fe at the highest magnetic field intensity. In it, the $U_{\text {eff }}$ values have the positive sign for undercoolings up to $\Delta T=100 \mathrm{~K}$ and experience a maximum of $U_{\text {eff }}=1.08 \mathrm{~m} / \mathrm{s}$ at $\Delta T=70 \mathrm{~K}$. The $U_{\text {eff }}$ values become negative for $\Delta T>$ $90 \mathrm{~K}$ and their magnitude increases with rising undercooling. The magnetic field intensity dependence of the $U_{\text {eff }}$ values for pure Ni is shown in Fig. 7b. The $U_{\text {eff }}$ values experience two negative maxima with rising magnetic field intensity. The first negative maximum occurs at $B=1 \mathrm{~T}$ for $\Delta T=30 \mathrm{~K}$. This critical magnetic field is shifted to $B=$ $3 \mathrm{~T}$ for $\Delta T=120 \mathrm{~K}$. The second negative maximum occurs at a magnetic field intensity of $B=5 \mathrm{~T}$ regardless of undercooling. It has smaller magnitude than that of the first one for $\Delta T=30 \sim 100 \mathrm{~K}$, but has larger magnitude for $\Delta T=110 \sim 120 \mathrm{~K}$. While this first negative maximum appears similar to that for pure Fe, the second negative maximum represents a difference between the two metals. It is unclear if a small hump observed at $B=4 \mathrm{~T}$ has a physical origin or not. Here we tentatively assume that it is due to the errors of the numerical calculations as well.

\section{Discussion}

The modelling shown in Section 4 unveiled similar dependences of the $U_{\text {eff }}$ values on undercooling and magnetic field intensity for pure Fe and pure Ni. The major difference between the two metals is the generally smaller magnitude of the $U_{\text {eff }}$ values for pure Fe. The modelling also showed that the transverse flow (see the flow types $\mathrm{b}$ and $\mathrm{c}$ of 
Fig. 1) dominates over a wide range of lower magnetics field intensities as evidenced by the depression in tip growth velocity and the corresponding negative sign of the $U_{\text {eff }}$ values. In contrast, the incident flow (generated by the flow type a of Fig. 1) becomes dominant at the highest magnetic field intensity of $B=6 \mathrm{~T}$ for small undercoolings as seen by the positive sign of the $U_{\text {eff }}$ values. The circular flow (see flow type a of Fig. 1) is dominant in a combined condition of the highest magnetic field intensity and high undercoolings as evidenced by the change of the sign from positive to negative. The circulation becomes so confined around the tip that it homogenises the local thermal gradient leading to coarsening of the tip. The undercooling and magnetic field intensity dependences of the $U_{\text {eff }}$ values corresponding to the transverse flow are discussed below first due to its overwhelming dominance in the range of magnetic field intensities explored.

As explained elsewhere [20], the transverse flow brings hot liquid from secondary arms of one primary trunk to the tip of another primary trunk of the same dendrite. To further understand the observed behavior, the following analytic approach is used to characterise the transverse flow. With a vanishing thermal gradient outside of the thermal boundary layer, $r$, the average volumetric thermoelectric force becomes

$$
\int_{0}^{r} \frac{\sigma S \nabla T B}{L / 2} d x=\frac{2 \sigma S \nabla T B r}{L}
$$

where the averaging is taken as half the characteristic length, $L$ due to the symmetry of the dendrite, $\sigma$ is the liquid thermal conductivity, $S$ is the difference in the absolute thermoelectric power between the solid and its liquid, $\nabla T$ is the thermal gradient inducing the TEMHD flow, and $r$ is the thermal diffusion distance in the liquid. Then by approximating the viscous force as $\mu \nabla^{2} U_{T E}=\mu U_{T E} / L^{2}$ and the electromagnetic damping force as $\sigma U_{T E} \times \boldsymbol{B} \times \boldsymbol{B}=\sigma U_{T E} B^{2}$, a steady-state balance between the 
thermoelectric, viscous and electromagnetic damping forces becomes

$$
\frac{2 \sigma S \nabla T B r}{L}=\frac{\mu U_{T E}}{L^{2}}+\sigma U_{T E} B^{2} .
$$

By substituting the approximations $r=D_{T} / V$ where $D_{T}$ is thermal diffusivity, $\nabla T=$ $\Delta T V / D_{T}$ and the Hartmann number $\mathrm{Ha}=B L(\sigma / \mu)^{1 / 2}$ into Eq. 2, the TEMHD flow velocity can be written as

$$
U_{T E}=\frac{2 S \Delta T}{\left(\frac{1}{H a}+H a\right)}\left(\frac{\sigma}{\mu}\right)^{\frac{1}{2}} .
$$

To calculate Ha, the characteristic length $L$ needs to be known. For this purpose, we define a critical magnetic field intensity of $B_{C}$, at which the reduced growth velocities shows the largest depression. At this critical intensity, it can be assumed that $\mathrm{Ha}=1$ [17] and Eq. (3) reduces to

$$
U_{T E}=S \Delta T\left(\frac{\sigma}{\mu}\right)^{\frac{1}{2}}
$$

As seen in Fig. 4, the reduced growth velocities of pure Fe show a clear minimum at $B$ $=2 \mathrm{~T}$ for all undercoolings, whereas those of pure Ni show a minimum at $B=1 \mathrm{~T}, 2 \mathrm{~T}$ and $3 \mathrm{~T}$ for $\Delta T=30 \mathrm{~K}, 80 \mathrm{~K}$ and $120 \mathrm{~K}$, respectively. For simplicity it is assumed that this characteristic length is held constant at any undercooling and therefore the critical magnetic field intensity can be taken as $B_{C}=2 \mathrm{~T}$. The observed dependences of the $U_{\text {eff }}$ values for the two metals as well as the differences between them can be understood in terms of Eq. (3).

First of all, the transverse flow velocity $U_{T E}$ is proportional to the undercooling $\Delta T$ and thus its magnitude will increase linearly with $\Delta T$ according to Eq. (3). Its magnetic field dependence is determined by the denominator of Eq. (3). The denominator varies nonlinearly with rising magnetic field intensity $B$. Thus, the dependency of the 
transverse flow velocity $U_{T E}$ on $B$ is also nonlinear. As listed in Table 1, the two metals studied have different material properties that affect this behavior. The values of $\sigma / \mu$ and $S$ of pure Ni are double those of pure Fe. From $\mathrm{Ha}=1$, its characteristic length scale $L$ is smaller than that for pure $\mathrm{Fe}$, if an averaged critical magnetic field of $B_{C}=2 \mathrm{~T}$ is taken for the reduced tip growth velocities of pure Fe. It is not certain what feature of the system this characteristic length represents. However, the morphological features of Fe dendrites should be generally larger than those of Ni dendrites as Fe dendrites have significantly larger tip radii (see Fig. 3b). By deriving Eq. (3) in such a form, Ha is equivalent for both $\mathrm{Fe}$ and $\mathrm{Ni}$ at the same magnetic field intensity. Therefore, the magnitude of the flow velocity $U_{T E}$ depends on the product of $S$ and $(\sigma / \mu)^{1 / 2}$ for a given undercooling. Using the material properties listed in Table 1, the product for pure $\mathrm{Ni}$ is about 2.7 times larger than that for pure Fe. For a quantitative comparison, we test two equivalent cases for the two metals. In the first case, the transverse flow velocities $U_{T E}$ calculated using Eq. (3) show a good agreement with the effective flow velocities $U_{\text {eff }}$ determined by the AG theory at the critical magnetic field of $B_{C}=2 \mathrm{~T}$ for each metal (see Fig. 8). This case was selected as it represents $\mathrm{Ha}=1$, which was a necessary assumption to obtain the characteristic length, though the origin of which is not well understood in the context of free dendritic growth. All other assumptions and material properties are independent of the experimental data. The second case is for $\Delta T=100 \mathrm{~K}$ for both $\mathrm{Fe}$ and $\mathrm{Ni}$. This case was selected as the relative effect of the transverse flow on the tip growth velocity $V$ should be lower at high undercoolings due to the power law dependences of $V$ on $\Delta T$ with an exponent larger than 1 (see Tables 2 and 3). The magnetic field may cause morphological changes, which in turn will alter the formation of thermoelectric currents, both of which may affect the characteristic length $L$. The results are shown in Fig. 8b.The predicted dependences of $U_{T E}$ on magnetic field 
intensity are again in good agreement with those of $U_{\text {eff }}$ shown in Figs. 6 and 7. The model achieves a consistent behavior across a wide range of undercooling and magnetic field intensity, and a comparable magnitude of the $U_{\text {eff }}$ values for both metals strongly suggesting that the source of this flow is indeed TEMHD. The model appears to give a poorer match to the experiments at high magnetic field intensities. The reason for this poorer match is not yet clear, but one must consider large uncertainties in the material properties, especially those in $S$, with at least $50 \%$ variability according to an early study [23]. There are additional phenomena that may be occurring but will not be captured by this simple theory. Previous numerical simulations [13-15] have shown that dendritic tips will lose their axial symmetry under the action of the transverse flow. This effect of the transverse flow will affect both this model and the AG theory, where the axis-symmetry of growing dendritic tips is assumed, leading to discrepancies in the $U_{\text {eff }}$ values.

The assumptions and calibrations in the application of Eq. (3) are valid for the transverse flow effect only. It was suggested that there could be multiple critical length scales, each of which may introduce a different type of fluid mechanism [17]. For example, at a very high magnetic field intensity the dominating flow can be the circular flow around the tip (see flow type a of Fig. 1). This circular flow may be characterised by the smaller length scale of the tip radius than that for the transverse flow. It is also unclear if the approximations used for the thermal gradient are still applicable to the incident flow and the circular flow. Previous numerical simulations [13-15] revealed that both the incident flow and the circular flow are localized in a very thin liquid layer close to the dendritic tip and arms. Therefore, their characteristic length scale is smaller than that assumed for the transverse flow. Accordingly the critical magnetic field intensity will be higher. However, the data of the reduced dendritic growth velocities 
shown in Fig. 4 do not allow us to specify the critical magnetic fields for neither flow type. Thus, it remains difficult to justify the magnitude of the $U_{\text {eff }}$ values calculated from the AG theory. Efforts are being made to develop a suitable model allowing for a quantitative description of $U_{T E}$ of the incident flow and the circular flow as a function of $\Delta T$ and $B$. It should be pointed out that it is also difficult to measure local flow velocities using the state-of-the-art techniques as in Thess et al. [31] because of small sizes of the glass-fluxed sample and short life time of TEMHD flows in solidification. Numerical microstructural simulations are under the way, which may provide a parameter-free justification of the calculated $U_{\text {eff }}$ values for the three kinds of TEMHD flows identified.

\section{Conclusions}

The dendritic growth velocities of pure Fe under static magnetic fields have been measured and compared with those obtained for pure Ni. The effective flow velocities due to TEMHD convection have been calculated by modelling of the measured data using the AG theory. A simple force-balance model has been proposed to justify the calculated effective flow velocities for the flow transverse to the growing tips. The conclusions drawn are as follows:

(1) The measured data of pure Fe without the magnetic field show smaller growth velocities than those obtained for pure $\mathrm{Ni}$ at all accessible undercoolings. This difference between the two metals can be ascribed to smaller thermal diffusivity and the smaller interfacial kinetic coefficient of pure Fe.

(2) The measured data of pure Fe with the magnetic fields shows a depression and a recovery of the growth velocities at the low magnetic field intensity of $B=1 \sim 3 \mathrm{~T}$ and the high magnetic field intensity of $B=4 \sim 6 \mathrm{~T}$, respectively. This magnetic field effect 
is similar to that observed for pure Ni. However, the recovery of the growth velocities of pure Fe is induced by a lower magnetic field intensity.

(3) The effective flow velocities at dendritic tips show similar kinds of undercooling and magnetic field intensity dependences for the two metals. The magnitude of the effective flow velocities for pure Fe is generally smaller than that for pure Ni. This difference between the two metals has been attributed to the differences in material properties, especially that of the absolute thermoelectric power between the solid and its liquid. The calculated effective flow velocities for the transverse flow show an agreement with the prediction of the force-balance model proposed based on assumption of a constant characteristic length. However, those for the incident flow and the circular flow dominating at the highest magnetic field intensity await justification with new models and parameter-free numerical simulations.

\section{Acknowledgement}

The authors thank Jiayun Cai, Jia Liu, Xuewei Zhao, Mengkun Han, Weina Zhao and Dandan Zhao for their assistance in the experimental work and image analyses. The authors also thank Jan Gegner, Song Cai and Zhu Zhai for providing the computer codes for image analysis and modelling. The authors are grateful to Peter Galenko, Dmitri Alexandrov and Christian Karrasch for helpful discussion. This work is financially

supported by the National Natural Science Foundation of China (51071043, 51211130113), Fundamental Research Funds for Central Universities (N09050901 and N130509001) and the International Exchanges Scheme of the Royal Society of the United Kingdom. 


\section{List of References}

[1] M. A. Jaworski, T. K. Gray, M. Antonelli, J. J. Kim, C. Y. Lau, B. M. Lee, M. J. Neumann, W. Xu, D. N. Ruzic, Thermoelectric magnetohydrodynamic stirring of liquid metals, Phys. Rev. Lett. 104 (2010) 094503.

[2] A. Kao, K. Pericleous, The effect of secondary arm growth on thermoelectric magnetohydrodynamics, Magnetohydrodynamics 48 (2012) 361-370.

[3] J. A. Shercliff, Thermoelectric magnetohydrodynamics, J. Fluid Mech. 91 (1979) $231-251$.

[4] L. A. Gorbunov, Effect of thermoelectromagnetic convection on the production of bulk single-crystals consisting of semiconductor melts in a constant magnetic field, Magnetohydrodynamics 23 (1987) 65-69.

[5] Y. M. Gel'Fgat, L. A. Gorbunov, An additional source of forced convection in semiconductor melts during single-crystal growth in magnetic fields, Sov. Phys. Dokl. 34 (1989) 470-473.

[6] T. Alboussiere, R. Moreau, D. Compte, Influence of a magnetic-field on the solidification of metallic alloys, Comptes Rendus de Lacademie des Sciences Serie II, 313 (1991) 749-755.

[7] Y. Y. Khine, J. S. Walker, F. R. Szofran, Themoelectric magnetohydrodynamic flow during crystal growth with a moderate or weak magnetic field, J. Cryst. Growth $212(2000) 584-596$.

[8] S. Yesilyurt, L. Vjusic, S. Motakef, F. R. Szofran, A. Croell, The influence of thermoelectromagnetic convection (TEMC) on the Bridghman growth of semiconductors, J. Cryst. Growth 211 (2000) 360-364.

[9] R. Moreau, O. Laskar, M. Tanaka, D. Camel, Thermoelectric magnetohydrodynamic effects on solidification of metallic alloys in the dendritic 
regime, Mater. Sci. Eng. A 173 (1993) 93-100.

[10] P. Lehmann, R. Moreau, D. Camel, R. Bolcato, A simple analysis of the effect of convection on the structure of the mushy zone in the case of horizontal Bridgman solidification: comparison with experimental results, J. Cryst. Growth 183 (1998) $690-704$.

[11] P. Lehmann, R. Moreau, D. Camel, R. Bolcato, Modification of interdendritic convection in directional solidification by a uniform magnetic field, Acta Mater. 46 (1998) 4067-4079.

[12] X. Li, Y. Fautrelle, Z. Ren, Influence of thermoelectric effects on the solid-liquid interface shape and cellular morphology in the mushy zone during the directional solidification of Al-Cu alloys under a magnetic field, Acta Mater. 55 (2007) $3803-3813$.

[13] X. Li, A. Gagnoud, Z. Ren, Y. Fautrelle, R. Moreau, Investigation of thermoelectric magnetic convection and its effect on solidification structure during directional solidification under a low axial magnetic field, Acta Mater. 57 (2009) 2180-2197.

[14] A. Kao, Thermoelectric magnetohydrodynamics in dendritic solidification, Doctoral Thesis of University Greenwich, United Kingdom, 2010.

[15] A. Kao, K. Pericleous, Investigating magnetic field orientation as an operational parameter in thermoelectric MHD solidification, J. Iron Steel Res. Inter. 19 (2012) $260-264$

[16] I. Kaldre, Y. Fautrelle, J. Etay, A. Bojarevics, L. Buligins, Thermoelectric current and magnetic field interaction influence on the structure of directionally solidified Sn-10wt\%Pb alloy, J. Alloys Compd. 571 (2013) 50-55.

[17] A. Kao, Analytic solutions to determine critical magnetic fields for thermoelectric magnetohydrodynamics in alloy solidification, Metall. Mater. Trans. A 46 (2015) 
4215-4233.

[18] J. Wang, Y. Fautrelle, H. Nguyen-Thi, G. Reinhart, H. L. Liao, X. Li, Y. B. Zhong, Z. M. Ren, Thermoelectric magnetohydrodynamic flows and their induced change of solid-liquid interface shape in static magnetic field-assisted directional solidification, Metall. Mater. Trans. 47 (2016) 1169-1179.

[19] A. Kao, B. Cai, P. D. Lee, K. Pericleous, The effects of thermoelectric magnetohydrodynamics in directional solidification under a transverse magnetic field, J. Cryst. Growth 457 (2017) 270-274.

[20] J. Gao, M. K. Han, A. Kao, K. Pericleous, D. V. Alexandrov, P. K. Galenko, Dendritic growth velocities in an undercooled melt of pure nickel under static magnetic fields: A test of theory with convection, Acta Mater. 103 (2016) 184-191.

[21] D. V. Alexandrov, P. K. Galenko, Selection criterion of stable dendritic growth at arbitrary Péclet numbers with convection, Phys. Rev. E 87 (2013) 062403.

[22] D. V. Alexandrov, P. K. Galenko, Dendrite growth under forced convection: analysis methods and experimental tests, Physics-Uspekhi 57 (2014) 771-786.

[23] J. E. Enderby, B. J. Dupree, The thermoelectric power of liquid Fe, Co and Ni. Philos. Magn. 35 (1977) 791-793.

[24] J. Gao, A. Kao, V. Bojarevics, K. Pericleous, P. K. Galenko, D. V. Alexandrov, Modelling of convection, temperature distribution and dendritic growth in glassfluxed nickel melts, J. Cryst. Growth 471 (2017) 66-72.

[25] J. W. Lum, D. M. Matson, M. C. Flemings, High-speed imaging and analysis of the solidification of undercooled nickel melts, Metall. Mater. Trans. B 27 (1996) $865-870$.

[26] S. R. Coriell, D. Turnbull, Relative roles of heat transport and interface rearrangement rates in the rapid growth of crystals in undercooled melts, Acta 
Metall. 30 (1982) 2135-2139.

[27] J. Monk, Y. Yang, M. I. Mendelev, M. Asta, J. J. Hoyt, D. Y. Sun, Determination of the crystal-melt interface kinetic coefficient from molecular dynamics simulations, Model. Simul. Mater. Sci. Eng. 18 (2010) 015004.

[28] J. Liu, R. L. Davichack, H. B. Dong, Molecular dynamics calculation of solidliquid interfacial free energy and its anisotropy during iron solidification, Comp. Mater. Sci. 74 (2013) 92-100.

[29] K. C. Mills, B. J. Monaghan, B. J. Keene, Thermal conductivities of molten metals: Part 1 pure metals, Int. Mater. Rev. 41 (1996) 209-242.

[30] T. Iida, I. L. Guthrie, The Physical Properties of Liquid Metals, Oxford Univ. Press, New York, 1988.

[31] A. Thess, E. V. Votyakov, Y. Kolesnikov, Lorentz force velocimetry, Phys. Rev. Lett. 96 (2006) 164501. 


\section{List of Figure Captions}

Fig. 1. Illustration of key features of TEMHD flow patterns due to magnetic fields perpendicular and transverse to the tip growth direction of an equiaxed dendrite (reproduced from Ref. 2). Note that (a), (b) and (c) are not all different flow types. (a) is the flow for a parallel magnetic field, (b) is the flow for a transverse magnetic field, and (c) highlights the transport of hot liquid to the tip that slows growth.

Fig. 2. Illustration of the method for determination of dendritic growth velocities from recorded video images. In each video image, numbers show distances and time travelled by a thermal front during recalescence of a glass-fluxed sample of pure Fe.

Fig. 3. (a) Measured dendritic growth velocities and (b) calculated dendritic tip radii $R$ of pure $\mathrm{Fe}$ and pure $\mathrm{Ni}$ without the static magnetic fields as a function of undercooling. The dendritic growth velocity data of pure Ni are taken from Ref. 20.

Fig. 4. Measured dendritic growth velocities of pure Fe under static magnetic fields as a function of undercooling. Lines represent power law fittings to the measured data.

Fig. 5. Reduced dendritic growth velocities of (a) pure Fe and (b) pure Ni as a function of magnetic field intensity. Numbers in (a) and (b) show undercoolings of the samples.

Fig. 6. Effective flow velocities calculated for pure Fe from the AG theory as a function of (a) undercooling and (b) magnetic field intensity. Numbers in (b) show undercoolings of liquid Fe. 
Fig. 7. Effective flow velocities for pure $\mathrm{Ni}$ as a function of (a) undercooling and (b) magnetic field intensity. Numbers in (b) show undercoolings of liquid Ni.

Fig. 8. Predicted values of the transverse flow velocities for pure $\mathrm{Fe}$ and pure $\mathrm{Ni}$ as a function of (a) undercooling and (b) magnetic field intensity. In (a), the magnetic field intensity is set to be $B=2 \mathrm{~T}$. In (b), the undercooling is set to be $\Delta T=100 \mathrm{~K}$. The effective flow velocities calculated from the AG theory are also shown for comparison. 